\title{
O conhecimento dos enfermeiros residentes sobre o cuidado à pessoa com estomia intestinal
}

\section{Resident nurses' knowledge of care for people with intestinal ostomy \\ El conocimiento de los enfermeros residentes sobre el cuidado de la persona con ostomía intestinal}

\author{
Roberto Corrêa Maffia Farias ${ }^{I}$; Norma Valéria Dantas de Oliveira Souza ${ }^{I I}$; Francisco Gleidson de Azevedo Gonçalves ${ }^{\text {III; }}$ \\ Helena Maria Scherlowski Leal David ${ }^{I V}$; Ariane da Silva Pires ${ }^{V}$; Luanna Klaren de Azevedo Amorim ${ }^{V I}$
}

\begin{abstract}
RESUMO: Objetivou-se analiar as potencialidades e fragilidades no conhecimento dos enfermeiros residentes acerca do cuidado ao cliente com estomia intestinal. Trata-se de uma pesquisa quantitativa e transversal, desenvolvida em hospital universitário no Município do Rio de Janeiro, no ano de 2012, com 35 residentes. Evidenciaram-se potencialidades (acertos) no conhecimento dos residentes de clínica cirúrgica, das quais se destacaram: localização e principais tipos de estomias (90\%); troca do sistema coletor e higienização do estoma (66\%); detecção de complicações e medidas preventivas (76\%); orientação à família $(27 \%)$. Ressaltaram-se fragilidades no conhecimento dos residentes de clínica médica para atuarem junto a estes clientes, tais como dificuldades em relação à orientação para o autocuidado (87\%) e detecção de complicações e medidas preventivas $(87 \%)$. Conclui-se que os residentes da clínica médica revelaram mais fragilidades no conhecimento sobre o tema do que os da clínica cirúrgica.

Palavras-Chave: Ostomia; enfermagem; ensino; educação em saúde.
\end{abstract}

ABSTRACT: This quantitative study aimed to examine the strengths and weaknesses in 35 resident nurses' knowledge of care for clients with ostomy was conducted at a university hospital in Rio de Janeiro city in 2012. Strengths (right answers) showed in the residents' surgical clinical knowledge, particularly of the location and main types of ostomy $(90 \%)$, replacing the collector system and cleaning the ostomy $(66 \%)$, detection of complications and preventive measures $(76 \%)$, and family guidance $(27 \%)$. Salient weaknesses in clinical medical residents' knowledge of working with these clients included difficulties regarding: selfcare guidance $(87 \%)$, and detection of complications and preventive measures $(87 \%)$. It was concluded that internal medicine residents displayed more weaknesses in knowledge of the subject than surgical clinical residents.

Keywords: Ostomy; nursing; teaching; health education.

RESUMEN: El objetivo del estudio fue analizar las potencialidades y debilidades en el conocimiento de los enfermeros residentes sobre la atención al cliente portador de ostomía intestinal. Se trata de una investigación cuantitativa y transversal, desarrollada en un hospital universitario en la ciudad de Rio de Janeiro, en 2012, junto a 35 residentes. Se han evidenciado potencialidades (aciertos) en el conocimiento de los residentes de clínica quirúrgica, de las que destacaron: ubicación y principales tipos de ostomías (90\%); cambio del sistema colector e higienización del estoma (66); detección de complicaciones y medidas preventivas $(76 \%)$; orientación a la familia $(27 \%)$. Se resaltan debilidades en el conocimiento de los residentes de la clínica médica para trabajar junto a esos clientes, tales como dificultades en cuanto a ofrecer orientación para el autocuidado (87\%) y detectar complicaciones y medidas preventivas $(87 \%)$. Se concluye que los residentes de clínica médica revelaron más debilidades en el conocimiento sobre el tema que los de la clínica quirúrgica.

Palabras Clave: Ostomía; enfermería; enseñanza; educación en salud.

\section{INTRODUÇÃO}

O objeto deste estudo é o conhecimento de enfermeiros residentes sobre o processo de cuidar/ cuidado de pessoas com estomia intestinal. $\mathrm{O}$ interesse pela temática ocorreu por meio da prática profissional como enfermeiro e docente de enfermagem em uma clínica cirúrgica de uma universidade do Município do Rio de Janeiro. Nessa oportunidade, observou-se, empiricamente, que muitos residentes de enfermagem apresentavam dificuldades no cuidado de pessoas com estomias intestinais, princi-

IEspecialista em Estomaterapia pela Faculdade de Enfermagem da Universidade do Rio de Janeiro. Especialista em Clínica Cirúrgica nos moldes de Residência em Enfermagem. Rio de Janeiro, Brasil. E-mail: betoenf@bol.com.br

IIDoutora em Enfermagem. Professora Associada do Departamento de Enfermagem Médico-Cirúrgica. Professora Permanente do Programa de Pós-Graduação da Faculdade de Enfermagem da Universidade do Estado do Rio de Janeiro. Brasil._E-mail: norval_souza@yahoo.com.br.

IIIMestre em Enfermagem pelo Programa de Pós-Graduação da Faculdade de Enfermagem da Universidade do Estado do Rio de Janeiro. Especialista em Enfermagem do Trabalho. Rio de Janeiro, Brasil.E-mail: gleydy_fran@hotmail.com.

IV Doutora em Saúde Coletiva. Professora Permanente do Programa de Pós-Graduação da Faculdade de Enfermagem da Universidade do Estado do Rio de Janeiro. Brasil. E-mail: helena.david@gmail.com.

${ }^{v}$ Doutoranda em Enfermagem. Especialista em Enfermagem em Estomaterapia. Professora Assistente do Departamento de Enfermagem Médico-Cirúrgica da Universidade do Estado do Rio de Janeiro. Brasil.E-mail: arianepires@oi.com.br.

viMestre em Enfermagem pelo Programa de Pós-Graduação da Faculdade de Enfermagem da Universidade do Estado do Rio de Janeiro. Brasil. E-mail: luklaren@yahoo.com.br. 
palmente no manejo dos estomas e nas orientações para o autocuidado.

Percebeu-se que alguns enfermeiros das unidades de internação demonstravam não só insegurança na atuação, mas também déficit teórico em relação à assistência ao cliente estomizado. Tais observações empíricas fortaleceram o desejo de investigar o objeto anteriormente apontado, especificamente entre os residentes, por estarem em processo formal de aprendizagem; portanto, estariam mais afeitos ao aprimoramento profissional.

Como contribuição, este estudo busca ampliar as discussões acerca da assistência aos clientes com estomia intestinal, sendo relevante, pois pode proporcionar subsídios para a elaboração de propostas que favoreçam a ampliação do conhecimento sobre o tema, auxiliando, desta forma, na capacitação do profissional.

A partir dessa breve contextualização acerca do objeto, selecionou-se o seguinte problema de pesquisa: os enfermeiros residentes das unidades de clínica cirúrgica e de clínica médica detêm conhecimento teórico para o processo de cuidar/cuidado do cliente com estomia intestinal?

De acordo com o problema de pesquisa, elencou-se um objetivo geral: avaliar o conhecimento do enfermeiro residente para a prestação do cuidado ao cliente com estomia intestinal. Nesta perspectiva, selecionou-se como objetivo específico: analisar as potencialidades e fragilidades no conhecimento dos enfermeiros residentes acerca do cuidado ao cliente com estomia intestinal.

\section{REVISÃO DE LITERATURA}

"O termo estomia tem origem grega e significa abertura artificial de um órgão interno na superfície do corpo criada cirurgicamente"1:336. Existem vários tipos de estomias, sendo as mais comuns, na prática clínica, as intestinais e as urinárias ${ }^{2}$.

Os estomas são nomeados a partir dos locais do corpo em que foram confeccionados. Assim sendo, as estomias intestinais podem ser denominadas de colostomias (situadas no cólon), ileostomias (confeccionadas no íleo) e jejunostomias (localizadas no jejuno) ${ }^{3}$.

A confecção de uma estomia é um acometimento impactante na vida de uma pessoa. Neste sentido, surge o sentimento de mutilação, o qual resulta na sensação de ser diferente das demais pessoas. Além disto, tal procedimento cirúrgico é gerador de ansiedade e temores, uma vez que está intimamente relacionado à autoimagem e ao convívio social, pois a pessoa perde a capacidade de continência das fezes e dos flatos, devendo viver dependente de um equipamento coletor que, muitas vezes, é incômodo e volumoso. Adiciona- do a tais transformações, verifica-se a dificuldade de inserção e manutenção no mundo do trabalho, pela necessidade de uso constante do banheiro e pelas restrições laborais próprias de quem possui um estoma ${ }^{3,4}$.

As principais doenças que levam à construção de estomas intestinais são: câncer colorretal, doença diverticular, doença inflamatória intestinal, incontinência anal, colite isquêmica, polipose adenomatose familiar, trauma, megacólon, infecções perineais graves, dentre outras ${ }^{5,6}$.

O enfermeiro é um dos primeiros integrantes da equipe multidisciplinar a se relacionar com o cliente estomizado no pós-operatório. Neste sentido, ele deve estar capacitado a responder às dúvidas e inquietações dessa clientela e a cuidar com segurança, prevenindo e detectando precocemente complicações que possam vir a prejudicar o processo de inclusão social ou comprometer a integridade biopsicossocial da pessoa estomizada ${ }^{7,8}$.

\section{Metodologia}

Estudo quantitativo, de desenho transversal, com variáveis obtidas por meio de um questionário pré-testado e validado por pares. $\mathrm{O}$ campo escolhido para o desenvolvimento da pesquisa foi um hospital universitário localizado no Município do Rio de Janeiro, onde se oferece assistência nos três níveis de atendimento (primário, secundário e terciário). Nessa instituição, são desenvolvidas atividades de ensino, pesquisa e extensão, servindo de campo prático para graduandos e pós-graduandos da área da saúde.

Os setores que serviram de campo de coleta de dados foram quatro enfermarias de clínica médica, juntamente com duas enfermarias de cirurgia geral, incluindo, também, a unidade intermediária cirúrgica. A escolha desses setores baseou-se no fato de serem lócus onde há clientes em pré e pós-operatório para confecção de estomas intestinais.

A população da pesquisa foi composta por enfermeiros residentes dos Programas de Clínica Cirúrgica e de Clínica Médica, do primeiro e do segundo anos de Residência, num total de 35 participantes. Foram excluídos residentes que atuassem com clientes estomizados há menos de três meses, o tempo mínimo para inclusão na pesquisa. Do mesmo modo, foram excluídos os residentes em férias e em licença médica no momento da coleta. Os excluídos totalizaram quatro participantes, ficando a população final com 31 residentes, sendo 19 de clínica médica e 12 de clínica cirúrgica.

A coleta de dados teve início após a autorização do Comitê de Ética em Pesquisa com Seres Humanos, por meio do parecer de $\mathrm{n}^{\mathrm{O}}$ 024.3.2012, respeitando, assim, a Resolução n ${ }^{\circ}$ 466/2012 do Conselho Nacional de Saúde (CNS). Antes de iniciar a coleta de dados com cada participante, um termo de consentimento 
livre e esclarecido era fornecido, explicando objeto, objetivos e cuidados quanto ao sigilo e ao anonimato dos participantes. Assinado o termo em duas vias, uma era entregue ao participante e a outra permanecia com os pesquisadores.

O instrumento de coleta de dados consistiu de um questionário com perguntas fechadas, composto por uma primeira parte de caracterização sociodemográfica dos participantes, e outra com perguntas que exploraram o conhecimento específico sobre o cuidado ao cliente com estomia intestinal.

O questionário foi elaborado pelos pesquisadores, sendo revisado e validado por dois peritos: um da área de metodologia da pesquisa e o outro do campo da estomaterapia. Além disso, realizaram-se pré-testes com o instrumento, aplicando-o a três enfermeiros residentes de outros programas de residência, diferentes dos de clínica médica e de cirúrgica, verificando-se a clareza na leitura e entendimento das questões. A coleta de dados ocorreu em julho e agosto de 2012.

$\mathrm{O}$ conteúdo do questionário privilegiou quatro temáticas consideradas relevantes para garantir um cuidado seguro e de qualidade: I) localização e principais tipos de estomias intestinais; II) cuidados durante a troca do sistema coletor e higienização do estoma; III) detecção de complicações e medidas de prevenção de alterações relacionadas aos estomas; e IV) orientação ao cliente/família voltada para o autocuidado.

Cada temática continha três perguntas fechadas com quatro alternativas, sendo apenas uma correta, perfazendo um total de 12 perguntas. Os resultados foram contabilizados em frequências simples e percentuais. A avaliação de desempenho dos participantes foi analisada de acordo com cada temática abordada no formulário de pesquisa.

Os dados obtidos foram digitados em banco de dados apropriado, criado a partir do Programa MS-Excel, em sua versão 2007 para MS-Windows XP, especificamente para esta finalidade. Após a alimentação deste banco de dados e conferência das informações, foram realizadas análises descritivas apenas de frequências simples para todas as variáveis trabalhadas. Os dados foram apresentados por meio de gráficos e analisados à luz do referencial teórico da estomaterapia.

\section{Resultados}

\section{Perfil dos participantes do estudo}

Os 31 participantes que compuseram a amostra foram caracterizados quanto aos aspectos sociodemográficos, levando em conta as seguintes variáveis: sexo, idade e naturalidade. Os resultados evidenciam que a maioria dos residentes era do sexo feminino $30(96,7 \%)$; com relação à idade dos participantes, houve maior frequência entre o intervalo etário de 22 a 27 anos, registrando-se o limite máximo de 32 anos e o menor, de 22 anos.

Houve predominância de residentes com naturalidade do Rio de Janeiro - 25(80,6\%) -, contudo verificou-se um residente proveniente de cada um dos seguintes Estados: Bahia (BA), Espírito Santo (ES), Goiás (GO), São Paulo (SP), Rio Grande do Sul (RS) e Rio Grande do Norte (RN); assim, 6(19,4\%) entrevistados eram de fora do Rio de Janeiro.

Referente ao Programa de Residência em que os participantes estavam inseridos, $19(61,2 \%)$ pertenciam ao Programa de Clínica e 12(38,8\%) ao Programa de Cirúrgica, observando-se um número expressivo de residentes do primeiro ano de residência - 20(64,6\%). A maioria dos residentes - 26(83,8\%) - possuía apenas a residência como atividade laboral.

Quanto à instituição formadora na graduação, a maioria dos participantes $17(54,1 \%)$ era graduada em instituições de ensino de caráter privado; em contraposição, $14(45,1 \%)$ residentes eram egressos de instituições públicas. Cabe destacar que 7(22,5\%) residentes já possuíam outras especializações na área de Enfermagem, dentre as quais se registraram estomaterapia, gestão em saúde, enfermagem do trabalho e gerontologia. Com relação ao tempo de formado, $26(83,9 \%)$ residentes tinham entre seis meses e dois anos de conclusão do curso de graduação.

Quanto à adesão à pesquisa por Programa da Residência, o menor índice de participantes que responderam ao questionário foram os residentes do segundo ano de clínica médica (78\%). Em relação aos demais residentes, houve $100 \%$ de participação.

\section{Potencialidades e fragilidades no conheci- mento dos residentes}

Com relação ao conhecimento acerca do cuidado ao cliente com estomia intestinal, foram tratados, separadamente, os resultados relativos aos enfermeiros residentes de clínica cirúrgica e de clínica médica, pois o conteúdo teórico desses Programas são diferenciados. Desse modo, analisa-se a seguir o desempenho de cada um desses grupos, distintamente.

Os residentes de clínica cirúrgica apresentaram uma maior potencialidade (acertos) em relação às quatro temáticas abordadas no questionário - I) localização e principais tipos de estomias intestinais; II) cuidados durante a troca do sistema coletor e higienização do estoma; III) detecção de complicações e medidas de prevenção de alterações relacionadas aos estomas; IV) orientação ao cliente/família voltada para o autocuidado -, se comparados aos residentes de clínica médica.

Assim, quanto à localização e principais tipos de estomias intestinais, 28(90,3\%) residentes de clínica cirúrgica responderam corretamente a este tema. Sobre o cuidado com a troca do sistema coletor e 
higienização do estoma, 20 (64,5\%) enfermeiros atingiram as respostas esperadas. Quanto à detecção de complicações e medidas de prevenção relacionadas às alterações dos estomas intestinais, houve 23(74,1\%) acertos; e referente às orientações ao cliente/família, o número de acertos totalizou 7(22,5\%).

A distribuição de acertos dos residentes de clínica médica apontou os resultados 9(32\%), 17(54\%), $5(18 \%)$ e 4(13\%) para as temáticas I, II, III, IV, respectivamente; assim, o quantitativo de acertos foi menor para esses residentes.

Com relação às fragilidades, constata-se, nas quatro temáticas investigadas, que os residentes de clínica médica apresentaram uma proporção maior de fragilidade, uma vez que o quantitativo de erros foi elevada, na proporção de 21(68\%), 14(45,1\%), $25(80,6 \%)$ e $28(90,3 \%)$, em cada tema analisado. Fica evidente que os residentes de clínica cirúrgica obtiveram menos respostas erradas, nas seguintes proporções: $3(9,6 \%), 10(32,2 \%), 7(22,5 \%)$ e $23(74,2 \%)$, em cada temática abordada no questionário.

\section{DiscUSSÃo}

A distribuição dos residentes conforme o sexo evidenciou que a maioria dos participantes foi do sexo feminino, sendo $97 \%$ mulheres e 3\% homens. Este resultado está em consonância com demais estudos realizados sobre a temática que evidenciam a predominância de mulheres na graduação em enfermagem, ou seja, a enfermagem como uma profissão essencialmente feminina ${ }^{9,10}$.

É necessário, então, considerar a influência de Florence Nightingale ao institucionalizar, na Inglaterra vitoriana (1862), uma profissão para as mulheres, pois elas eram naturalmente preparadas, a partir de valores que se consideravam femininos naquela época ${ }^{11}$. Segundo o Instituto Nacional de Estudos e Pesquisas Educacionais (INEP), outra justificativa para esse resultado refere-se ao aumento da participação majoritariamente feminina, de 2001 a 2010, no ensino superior: em 2010, do total de 6.379.299 de matrículas no ensino superior, $57 \%$ eram mulheres e, entre os concluintes, a participação feminina atingiu $60,9 \%{ }^{12}$.

Em relação à faixa etária, $80 \%$ dos residentes pesquisados tinha idades entre 22 e 27 anos, seguidos de $20 \%$ dos residentes na faixa etária entre 28 e 32 anos. Esse resultado aproxima-se dos dados do Censo da Educação Superior (CES), realizado pelo INEP em 2009, segundo os quais a educação superior brasileira é predominantemente formada por pessoas do sexo feminino, com idade em média de 21 anos para vínculos de matrícula, 19 anos para forma de ingresso e 23 anos para a conclusão do curso de graduaçãa ${ }^{13}$.

Sobre a naturalidade, $65 \%$ dos residentes eram oriundos do Rio de Janeiro e 35\% tinham origem em outros Estados, como Bahia, Espírito Santo, Goiás, São Paulo, Rio Grande do Sul e Rio Grande do Norte. Essa diversificação de profissionais de enfermagem oriundos de outros estados confirma o reconhecimento nacional dos programas de residência do hospital universitário investigado, cuja missão é prestar assistência integrada, humanizada e de excelência à saúde, sendo agente transformador da sociedade por intermédio do ensino, da pesquisa e da extensão ${ }^{14}$.

Outro aspecto a ser considerado é o fato de a residência em enfermagem do presente estudo ter sido vinculada, no ano de 2011, à Faculdade de Enfermagem da Universidade, a qual pertence o hospital investigado. Desse modo, a residência passou a ser reconhecida como curso de especialização, o que vem garantir ao residente, no final do Programa, o título de especialista em determinada área de saber ${ }^{14}$.

Quanto à origem das instituições de ensino superior em que os residentes cursaram a graduação, observa-se um quantitativo prevalente oriundo de universidades privadas (52\%), em relação aos egressos de instituições públicas (48\%). Esse resultado está em consonância com os dados do Censo de Ensino Superior de 2010, segundo os quais a rede privada de ensino teve um grande crescimento desde 2001. A comprovação veio a partir do número de matrículas nessas instituições, que aumentou 59\% em detrimento dos $17 \%$ da rede pública, durante o mesmo período.

Em relação ao quantitativo de instituições de ensino superior, em 2010, havia 2.378 instituições, das quais $88,3 \%$ eram privadas, $4,5 \%$ estaduais, $4,2 \%$ federais e 3,0\% municipais. Assim, a oferta de vagas é consideravelmente menor nas instituições públicas, fato também verificado para o curso de enfermagem ${ }^{12}$.

Segundo o INEP, em 2010, ingressaram 2.182.229 alunos de graduação, o que corresponde a um aumento de 109,2\% em relação a 2001. Nesse período, a categoria federal apresentava, proporcionalmente, a maior elevação do número de ingressos (140,5\%), seguida da categoria privada $(115,4 \%)$. Por conseguinte, no ano de 2010, as instituições privadas contavam com 78,2\% dos ingressos de graduação; seguidas das federais, com 13,9\%; estaduais, com 6,5\%; e municipais, com 1,5\%. Ainda, o ano de 2010 apresentou 973.830 concluintes, representando o resultado mais expressivo; comparativamente aos demais anos, esse total é 145,8\% maior que o registrado em 2001. Destaque para a evolução ascendente da participação da categoria privada que, em 2010, foi responsável por $80,4 \%$ dos concluintes ${ }^{13}$.

Sobre o ensino superior no Brasil, em 2003, houve um aumento de $70 \%$ dos cursos, no setor privado, e apenas 30\% no setor público. Para o Curso de Graduação em Enfermagem, a oferta do setor privado era de $73,4 \%$, enquanto a de vagas chegou a ser de $88,7 \%$. A maioria dos cursos está localizada nas Regiões Sudeste e Sul, que detêm o maior PIB do país, 
fator determinante para o desenvolvimento da região. A maior proporção de profissionais de enfermagem está na Região Sudeste, o que expressa um mercado promissor, pois melhora o acesso para o ingresso nos cursos de graduação em enfermagem ${ }^{15}$.

Quanto ao tempo de formação dos enfermeiros residentes, nota-se que a maior parte era composta de recém-formados (83\%), possuindo em média entre seis meses e até dois anos de conclusão da graduação. Essa situação é explicada pela busca inicial do recém-formado pela capacitação e qualificação profissional na área de seu interesse. Nesse sentido, a escolha pela residência envolve a busca de saberes e práticas especializados para o estabelecimento de cuidado técnico, humano e ético. Desse modo, qualifica-se o enfermeiro para realizar atividades de educação em saúde, gerenciamento da assistência, ensino, pesquisa, extensão e oferta de cuidados em área específica, fornecendo-lhe título de especialista ${ }^{10}$.

Em relação aos residentes que possuíam outro vínculo empregatício, neste estudo, $16 \%$ realizam atividades laborais em concomitância com a residência e $84 \%$ deles desenvolviam apenas a referida especialidade. Isso pode ser explicado pelo fato de os programas de residência possuírem uma carga horária de 60 horas semanais, o que, na maioria das vezes, impossibilita o enfermeiro conciliar as atividades da residência com outras atividades laborais ${ }^{10}$.

$\mathrm{O}$ quantitativo de residentes que acumulava outra atividade laboral diminuiu no ano de 2013, pois o regimento do concurso para residência em enfermagem estabeleceu que o candidato matriculado devia se comprometer com o programa sob a forma de dedicação exclusiva. Essa exigência está em conformidade com as normas da Comissão Nacional de Residência Multiprofissional em Saúde e do Regimento Único dos Programas de Residência Multiprofissional e em Área Profissional da Saúde da Universidade do Estado do Rio de Janeiro ${ }^{14}$.

Os enfermeiros residentes de clínica médica apresentaram maior fragilidade em relação às quatro temáticas abordadas no estudo em comparação aos de clínica cirúrgica. Tal resultado pode ser justificado pela ausência de conteúdo teórico sobre estomias intestinais na programação teórica específica daquele Programa. Em contrapartida, na programação de clínica cirúrgica, os residentes constroem esse conhecimento, pois há aulas teóricas que visam preparar o residente para o processo de cuidar/cuidado a clientes em situação de estomia. Vale registrar que as enfermarias de clínica médica não são campos específicos para a admissão de clientes com estomias intestinais; porém, devido ao crescente acometimento de pessoas com problemas de saúde que levam à construção de estomas, verifica-se, então, um número cada vez maior de pessoas com estomias internadas nas enfermarias de clínica médica ${ }^{16}$.

A fragilidade de conhecimento mais prevalente dos residentes de clínica cirúrgica envolveu a temática IV orientação para o autocuidado. Esse conteúdo é mui- to importante, pois um dos objetivos da assistência ao cliente com estomia é capacitá-lo para o autocuidado a fim de que ele seja independente nas suas ações de vida diária. Ao aprender a se cuidar, o estomizado pode não só viver com mais qualidade de vida, mas também identificar, precocemente, alterações no seu processo saúde-doença, minimizando ou impedindo que complicações sejam instaladas ${ }^{7,17,18}$.

Em relação à maior prevalência de fragilidade teórica dos residentes de clínica médica, destaca-se a orientação para o autocuidado (87\%), seguida de perto pela temática III - diagnóstico das complicações e medidas preventivas para prevenção de alterações (82\%). Cabe enfatizar a importância da detecção precoce das complicações das estomias, as quais são muito diversificadas, como, por exemplo: a dermatite periestoma, o prolapso e a retração do estoma, a hérnia paraestomal e periestomal, a estenose de estoma, o descolamento mucocutâneo. Enfim, descrevem-se muitas complicações que retardam ou retrocedem o processo de inclusão social dos estomizados ${ }^{17,19}$.

Por esta razão, o enfermeiro precisa estar apto para detectar precocemente tais complicações, bem como para prescrever, realizar e supervisionar medidas preventivas e curativas que impeçam ou minimizem as complicações. Além disso, faz-se relevante salientar que a instalação de uma complicação pode gerar outra, como a retração do estoma favorecer a ocorrência da dermatite periestoma. Desse modo, corre-se o risco de aumentar o tempo de internação do cliente, ampliar o sofrimento biopsicossocial da pessoa e adicionar maior ônus financeiro para a pessoa, família e sociedade ${ }^{17}$.

Vale mencionar a ocorrência significativa de fragilidade do conhecimento deles na temática II cuidados durante a troca do sistema coletor e higienização do estoma, para residentes de clínica médica e cirúrgica. Por exemplo, preconiza-se diminuir as trocas da placa adesiva e prescreve-se limpeza da bolsa coletora e do estoma, o que envolve o uso de tecnologias que ajudam no bem-estar do cliente e, ao mesmo tempo, previnem complicações; há tecnologias que minimizam odores e permitem, em concomitância, a eliminação de flatos; prescrevem-se equipamentos coletores específicos para cada tipo de estoma, considerando também as características biopsicossociais do cliente. Neste sentido, há um saber relevante que o enfermeiro deve desenvolver para cuidar com segurança e qualidade da clientela estomizadas, garantindo bem-estar ${ }^{17}$.

\section{Conclusão}

Conclui-se que os residentes da clínica médica revelaram maior fragilidade no conhecimento sobre o tema do que os da clínica cirúrgica. No entanto, cabe enfatizar que ambos os grupos de residentes apresentaram fragilidades no saber relacionado ao cuidado à 
pessoa com estomia, as quais devem ser superadas, pois é preciso garantir uma assistência segura a esta clientela.

Considerando as fragilidades apresentadas pelos residentes, algumas considerações devem ser tecidas, entre as quais se destaca aquela que considera o conhecimento como dinâmico, principalmente na área da saúde, em que o quadro epidemiológico da população muda; também deve-se entender que as tecnologias podem ser utilizadas em favor da melhor assistência à clientela. Assim, relevando esses dois aspectos, há de se avaliar e adequar continuamente os currículos e programas teórico-práticos dos enfermeiros residentes a fim de capacitá-los para a realidade que os rodeiam.

Uma das limitações do presente estudo refere-se ao delineamento da pesquisa quantitativa, realizado em um único cenário, não permitindo generalizações. Recomenda-se a inclusão do tema nos currículos dos cursos de graduação, considerando esta uma séria problemática de saúde que merece atenção, a fim de garantir uma formação qualificada. Sugere-se a realização de novos estudos, em outros cenários e contextos, considerando o crescente número de estomizados, especialmente entre a população jovem.

\section{REFERÊNCIAS}

1.Bellato R, Pereira WR, Maruyama SAT, Oliveira PC. A convergência cuidado-educação-politicidade: um desafio a ser enfrentado pelos profissionais na garantia aos direitos à saúde das pessoas portadoras de estomias. Texto contexto - enferm. 2006; 15:334-42.

2.Oliveira D. O estigma da marca corporal invisível: estudo sobre o mundo do trabalho das pessoas com estomia intestinal definitiva [dissertação de mestrado]. Brasília (DF): Universidade de Brasília; 2007.

3.Santos VLCG. A estomaterapia através dos tempos. In: Santos VLCG, Cesaretti IUR, organizadores. Assistência em estomaterapia: cuidando do ostomizado. São Paulo: Atheneu; 2005. p. 1-17.

4.Ardigo FS, Amante LN. Conhecimento do profissional acerca do cuidado de enfermagem à pessoa com estomia intestinal e família. Texto contexto - enferm. 2013; 22:1064-71.

5.Habr-Gama A, Araújo AEA. Estomas Intestinais: aspectos conceituais e técnicos. In: Santos VLCG, Cesaretti IUR, organizadores. Assistência em estomaterapia: cuidando do ostomizado. São Paulo: Atheneu; 2005. p. 39-54.
6.Poletto D, Silva DMGV. Viver com estoma intestinal: a construção da autonomia para o cuidado. Rev Latino-Am Enfermagem. 2013; 21:531-8.

7.Maurício VC, Souza NVDO, Lisboa MTL. O enfermeiro e sua participação no processo de reabilitação da pessoa com estoma. Esc Anna Nery. 2013; 17:416-22.

8.Maurício VC, Souza NVDO, Lisboa MTL. Determinantes biopsicossociais do processo de inclusão laboral da pessoa estomizada. Rev Bras Enferm. 2014; 67:415-21. 9.Franco GP, Barros ALBL, Nogueira-Martins LA, Zeitoun SS. Burnout em residentes de enfermagem. Rev esc enferm USP. 2011; 45:12-8.

10.Goulart CT, Silva RM, Bolzan MEO, Guido LA. Perfil sociodemográfico e acadêmico dos residentes multiprofissionais de uma universidade pública. Rev Rene. 2012; $13: 178-86$

11.Lopes MJM, Leal SMC. A feminização persistente na qualificação profissional da enfermagem brasileira. Cad Pagu. 2005; 24:105-25.

12.Instituto Nacional de Estudos e Pesquisas Educacionais Anísio Teixeira (INEP). Ministério da Educação. Censo da Educação Superior 2010: divulgação dos principais resultados do censo da educação superior 2010. Brasília (DF): Ministério da Educação; 2011.

13.Instituto Nacional de Estudos e Pesquisas Educacionais Anísio Teixeira (INEP). Ministério da Educação. Censo da Educação Superior 2009: resumo técnico. Brasília (DF): Ministério da Educação; 2010.

14.Universidade do Estado do Rio de Janeiro. Deliberação n 26/2012. Autoriza a Criação do Curso de Especialização em Enfermagem na modalidade Residência. Rio de Janeiro: Universidade do Estado do Rio de Janeiro; 2012. 15.Galleguillos TGB, Catani AM. Avaliação da educação superior no Brasil e a expansão da educação superior em enfermagem. Educ e pesq. 2011; 37:843-60.

16.Mauricio VC. A pessoa estomizada e o processo de inclusão no trabalho: contribuição para enfermagem [dissertação de mestrado]. Rio de Janeiro: Universidade do Estado do Rio de Janeiro; 2011.

17.Aguiar ESS, Santos AAR, Soares MJGO, Ancelmo MNS, Santos SR. Complicações do estoma e pele periestoma em pacientes com estomas intestinais. Rev Estima. 2011; 9:22-30.

18.Souza NVDO, Santos NS, Penna LHG, Sampaio CEP, Melo CV, Leite GFP, Maurício VC. Avaliar para melhorar: perspectiva de discentes na avaliação do curso de extensão sobre estomias. Rev enferm UERJ. 2012; 20:235-41.

19.Silva AC, Silva GNS, Cunha RR. Caracterização de pessoas estomizadas atendidas em consulta de enfermagem do serviço de estomaterapia do Município de Belém-PA. Rev Estima. 2012; 10(1):12-9. 